\title{
SOCIAL ANXIETY AND SELF-REPORTED TIME SPENT ONLINE IN A SAMPLE OF ALBANIAN UNIVERSITY STUDENTS
}

\author{
Erika Melonashi ${ }^{1}$
}

\begin{abstract}
Online activity serves different purposes, one of them being communication and social interaction. Studies have demonstrated that individuals tend to display online behavioral patterns that are similar to their social groups; also there is some evidence that individuals who have interaction difficulties in real life (e.g., social anxiety) might engage more in online behavior. The purpose of the present study was to investigate the relationship between self-reported time spent online, descriptive social norms, and social anxiety symptoms in a sample of 356 Albanian University students. Participants were recruited online. Results showed a significant predictive model for self-reported time spent online, $\mathrm{F}(7,342)=48.99, \mathrm{p}<.001$, $\mathrm{R}^{2}=.50$. Age, gender, and the four social anxiety components were not significant predictors; only descriptive norms had a significant effect, $\beta=.70, p<.001$. Results are in line with the social normative approach to internet use and have several implications which are discussed in the paper.
\end{abstract}

UDC Classification: 159.9; DOI: http://dx.doi.org/10.12955/cbup.v5.1013

Keywords: Albania, social anxiety, online, students

\section{Introduction}

The widespread use of the internet has affected the dynamics of social relationships and communication patterns especially among youth. The excessive time spent online particularly during this developmental stage, represents a growing concern, both in terms of its impact on quality of life (interference with everyday functioning) and the greater risk for addiction. Hence it is important to investigate patterns of internet use, particularly in regard to the specific functions it fulfills during this developmental stage.

In the recent years, internet use in Albania has become quite widespread among different age groups. This phenomenon has been accompanied by growing concerns regarding negative consequences such as, gaming addiction, online sexual harassment, view of inappropriate content (for children) etc. (World Vision, 2014). Nonetheless, research on internet use patterns is still in its infancy as data comes mainly from organizations such as UNICEF and World Vision and focuses particularly on children and adolescents. So far, no research has been conducted among young adults, and also there has been no examination of theoretical constructs related to the specific behavior (in addition to frequency and prevalence data).

The present study aimed to assess the relationship between self-reported time spent online, social anxiety symptoms and descriptive norms among Albanian University students. Hence the study would determine whether a social normative approach or a stress-response/self-medication model, would better explain self-reported time spent online.

\section{Literature Review}

Internet use patterns are related to very diverse functions, including communication and social relationships, entertainment, stress management etc. According to a social normative approach of internet use, internet use patterns of youth are highly similar to their social groups, friends and peers (Chang, 2004; Zhou \& Fang, 2015). Thus, internet use patterns among youth might be explicable through processes such as social learning/modelling and group belonging, as well as factors such as descriptive or injunctive norms for internet use (how widespread and acceptable the behavior is) (see Brechwald \& Prinstein, 2011; Steinberg \& Monahan, 2007).

Yet another line of research focuses on an alternative function of internet use, e.g., as a stress management or anxiety relieving tool (e.g., Koc \& Gulyagci, 2013; Liberatore et al. 2011; Morrison \& Gore, 2012). There is some evidence that individuals who have difficulties in forming relationships in real life might turn to the online medium as a safer environment to experiment, approach others, and build new social connections (Joinson, 2001; Weinstein et al. 2015). In this context, several studies have reported relationships between social anxiety symptoms and internet addictive behavior (Yen et al., 2007; Wei et al. 2012).

\footnotetext{
${ }^{1}$ Faculty of Social Sciences and Education, European University of Tirana, erika.melonashi@uet.edu.al
} 
Social anxiety is a psychological disorder characterized by constant anxiety/fear as well as avoidance of social interaction or performance situations; most important, the presence of anxiety and avoidance behaviors interfere with normal functioning of the person (American Psychiatric Association, 2013). Data from the United States have reported that most college students experience at least some of social anxiety symptoms, although they do not fulfill the necessary criteria for diagnosis of the disorder (Baum et al., 2001; Bryan, 2005). Indeed, young adulthood poses significant developmental challenges on the individual in terms of achievement, acceptance, belonging, intimacy etc. (Erikson, 1978). Moreover, several studies with college students have reported relationships between social anxiety symptoms and problematic internet use suggesting that the online medium might serve as a safe place to experiment, form new social relationships, and relieve stress (Alavi et al., 2010; Liu \& Kuo, 2007; Montag et al., 2011).

To summarize, internet use patterns among youth might be explicable both in terms of social normative approaches (descriptive and injunctive social norms) and self-medication models (e.g., to manage social anxiety symptoms).

\section{Methodology}

\section{Participants}

Participants were 356 Albanian university students, 115 men and 241 women, Mean $_{\text {age }}=24.7$ years, $\mathrm{SD}=7.8$ years. In terms of field of study, participants were classified as follows: 112 students from the Faculty of Law, 133 students from the Faculty of Economics \& Information Technology and 111 students from the Faculty of Social Sciences.

\section{Procedure}

The study was conducted online as emails were sent to 500 email addresses of students randomly chosen from the institutional database. The content of the email explained the purpose of the study, ensured the anonymity and confidentiality of the respondents and provided the contact address of the researcher for further questions/clarifications. Students were asked to access the questionnaire through the link contained in the email (Google Docs Platform).

\section{Instruments}

The instrument was a self-report questionnaire with 3 main sections. The first section gathered information on demographics (gender, age, education profile). The second section included five questions referring to the time spent online (number of hours per day) of the participant, their best friend, the peer group, partner, and sibling. The response options were as follows: '1-2 hours', '3-4 hours', '5-6 hours' and 'more than 6 hours'. The four items referring to reports of best friend, peer group, partner and sibling were grouped into the variable 'Descriptive norm for online behavior.' The internal consistency for these items, Chronbach's alpha held an acceptable value of $\alpha=.73$, allowing the use of these items as a subscale.

The third section of the questionnaire was the Liebowitz Social Anxiety Scale (Liebowitz, 1987), an instrument consisting of 24 items, 11 referring to Social interaction situations and 13 to Performance situations. This scale asks participants to answer the questions twice, once for anxiety levels in the specific situations and once for avoidance behavior in the same situations. Response options were from 0 (None) to 3 (Severe) for Anxiety, and from 0 (Never) to 3 (Usually) for Avoidance. The total score of the scale has a maximum of 144 points, and the categories are as follows: Up to a score of 30, social anxiety is unlikely; Up to a score of 60 , it is probable; Up to 90 it is very probable, while higher than 90, highly probable. The internal consistency for Liebowitz scale as a whole was Chronbach's $\alpha$ $=.94$. The four subscales also had acceptable internal consistency as follows, Anxiety in social interaction $\alpha=.86$, Anxiety in performance situations, $\alpha=.84$, Avoidance of social interactions $\alpha=.84$, Avoidance of performance situations $\alpha=.79$.

The first and second sections of the instrument were constructed in Albanian, while the Liebowitz scale was translated from English. To ensure that the translation process was correct, the scale was translated by two independent translators and Albanian versions were compared by the researcher. In the few cases of word mismatch, the researcher (proficient in English), chose the 'most appropriate' item. This pre-final version was piloted with 5 students, to ensure the comprehensibility of the items. No major corrections were done after this stage. 


\section{Results}

Descriptive analyses revealed that students reported a mean time spent online of 2 hours/day $(\mathrm{M}=2.00$, $\mathrm{SD}=.89$ ), whereas the mean time reported for others, i.e., descriptive norm, was $\mathrm{M}=2.40$ hours/day, $\mathrm{SD}=.75$. As regards social anxiety, $21.3 \%$ of the sample (76 participants) reported total scores up to 30, i.e., social anxiety was unlikely for almost $1 / 5$ of the total sample. Additionally, $43.5 \%$ of the sample (155 participants) reported total scores up to 60, falling thus into the category of probable social anxiety. Finally, 30.1\% (107 participants) reported scores up to 90, falling into the category of very probable social anxiety and only $5.1 \%$ (18 participants) reported the highest scores in the scale (i.e., social anxiety was highly probable in this fraction of the sample). Descriptive statistics for total scores as well as the specific dimensions are shown in Table 1.

Correlation analyses between the variables of interest revealed significant relationships only between time reported online and descriptive norms for online behavior, $r=.70, p<.01$. The four dimensions of social anxiety as well as the total score had no significant correlations to time spent online. An interesting finding involved correlations between descriptive norms and three of the dimensions of social anxiety, respectively with Fear of social interaction, $r=-.12, \mathrm{p}<.05$, Avoidance of social interaction, $\mathrm{r}=-.16, \mathrm{p}<.01$, and Avoidance of performance, $\mathrm{r}=-.11, \mathrm{p}<.05$. Table 2 shows correlations between the variables of interest.

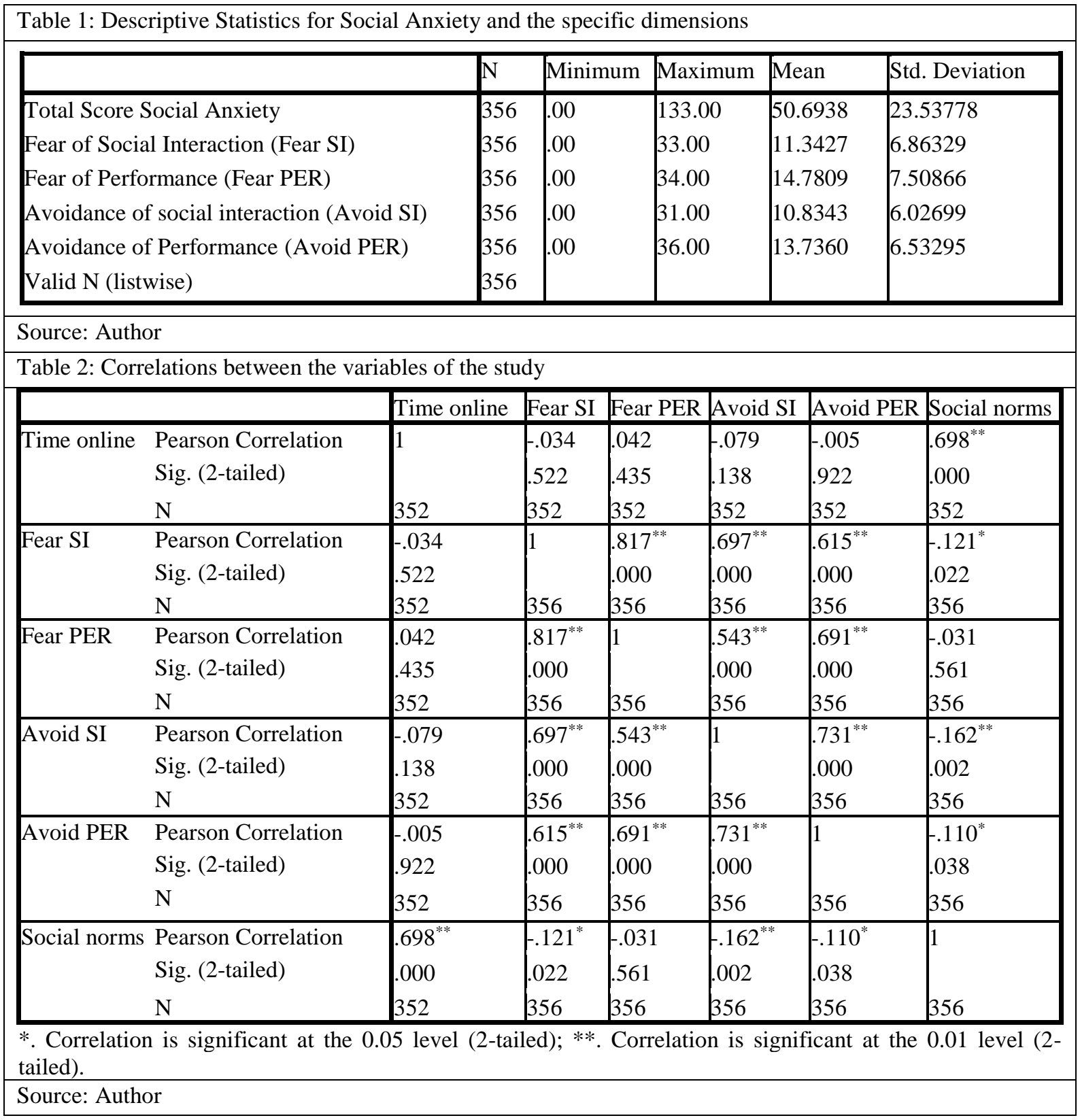


Subsequently, a regression analysis was conducted with time spent online as the dependent variable, and gender, age, descriptive norms and the four dimensions of social anxiety as independent variables. Results showed a significant predictive model for self-reported time spent online, F $(7,342)=48.99$, $\mathrm{p}<.001, \mathrm{R}^{2}=.50$. However, age, gender, and the four social anxiety components were not significant predictors; only the variable descriptive norms contributed significantly to the model, $\beta=.70, p<.001$.

\section{Discussion and Conclusions}

The present study aimed to assess the relationships between self-reported time spent online, social anxiety and descriptive social norms. Results demonstrated no significant relationships between social anxiety (as a total and also specific subscales) and self-reported time spent online. The only significant influence on time spent online, was that of descriptive social norms.

These results are in line with a social influence approach to explaining online behavior; indeed, participants reported that the online behaviors of their partners, peers, friends, siblings were highly similar to theirs. These results are in line with studies on adolescent internet use (e.g., Chang, 2004; Zhou \& Fang, 2015) suggesting that similar to that developmental stage, social influence in young adulthood might still be highly relevant in understanding online behavior.

Conversely, these findings might also highlight once more the positive function of the online medium as facilitator of social interaction and relationship maintenance (Young, 2015; Zhou \& Fang, 2015). Indeed, a very interesting finding was the negative relationship between descriptive social norms and three of the dimensions of social anxiety. More specifically participants who reported a greater involvement in online behavior of their social groups also reported lower levels of fear or avoidance of social interaction as well as less performance avoidance. This finding is in line with research suggesting the positive social supportive effects of online communication; for instance, Wangberg et al. (2007) have reported relationships between internet use and social support (social provision) and also subjective health. Nonetheless, the present study did not investigate the purpose/function of being online, (i.e., communication, work, information seeking etc.), an aspect which is very important to consider in future research (see Pontes, Szabo, \& Griffiths, 2015).

Another important aspect refers to excessive or problematic internet use, which certainly cannot be assessed through time spent online only (Lehenbaue-Baum \& Fohringer, 2015). Indeed, several studies have found relationships between internet addiction and social anxiety, suggesting that the relationship might become evident at more severe levels of problematic behavior (Alavi et al. 2010; Montag et al., 2011). Further research is needed to examine whether this is actually the case among Albanian youth, using measures of internet addiction apart from social anxiety measures.

Nonetheless, the present study also provides some other findings worth mentioning, especially in terms of social anxiety. For instance, descriptive analyses indicated that only $1 / 5$ of the sample fell into the category of 'unlikely social anxiety,' while the rest ranged from 'probable' to 'highly probable social anxiety.' Although the sample is too small and limited to conclude on prevalence rates, this data suggests the need for further research on social anxiety especially among Albanian youth. The socioeconomic and political transition of Albania in the last 27 years, as well as poverty, crime, corruption, job insecurity, the dissolution of the traditional family, the instability of values and social norms, are only some of the macro-level factors, which might suggest the relevance of investigating further disorders such as social anxiety. In support of this claim, Hofmann, Asnaani, and Hinton (2011) have suggested that the cultural context is very important in understanding a disorder such as social anxiety and have also reported significant differences in rates of the disorder across countries. Hence future research with more extensive and representative samples might be needed to examine this aspect also in Albania.

To conclude, the present study suggests that perceived social norms are relevant in understanding internet use behavior (time spent online) among Albanian students. Social anxiety with its' two components Fear and Avoidance, was not related to self-reported time spent online; also, other factors such as age and gender were irrelevant. Despite the several limitations, the findings of the present study have important implications in terms of future research within this field in Albania. 


\section{Acknowledgement}

This publication was made possible through the financial support of the European Institute "Pashko" of the European Education Initiative (EEI).

\section{References}

Alavi, S. S., Alaghemandan, H., Maracy, M. R., Jannatifard, F., Eslami, M. \& Ferdosi, M. (2010). Impact of addiction to Internet on a number of psychiatric symptoms in students of Isfahan universities. International Journal of Preventive Medicine, 3, 122-127.

American Psychiatric Association, (2013). Diagnostic and statistical manual of mental disorders (5th Ed.). USA: APA

Brechwald, W. A., \& Prinstein, M. J. (2011). Beyond homophily: A decade of advances in understanding peer influence processes. Journal of Research on Adolescence, 21, 166-179.

Chang, L. (2004). The role of classroom norms in contextualizing the relations of children's social behaviors to peer acceptance, Developmental Psychology, 40, 691-702.

Erikson, E. (1978). Childhood and Society. Great Britain: W. W. Norton \& Co.

Hofmann, S. G., Asnaani, A., \& Hinton, D. E. (2011). Cultural aspects in social anxiety and social anxiety disorder. Depress Anxiety, 27(12), 1117-1127.

Joinson, A. N. (2001). Self-disclosure in computer mediated communication: the role of self-awareness and visual anonymity. European Journal of Social Psychology, 31, 177-192.

Koc, M. \& Gulyagci, S. (2013). Facebook addiction among Turkish college students: The role of psychological health, demographic, and usage characteristics. Cyberpsychology, Behavior, and Social Networking, 16(4), 279-284.

Lehenbaue-Baum, M., \& Fohringer, M. (2015). Towards classification criteria for internet gaming disorder: Debunking differences between addiction and high engagement in a German sample of World of Warcraft players. Computers in Human Behavior, 45, 345-351.

Liberatore, K. A., Rosario, K., Colon-De Marti, L. N., \& Martinez. K. G. (2011). Prevalence of Internet addiction in Latino adolescents with psychiatric diagnosis. Cyberpsychology, Behavior, and Social Networking, 14, 399-402.

Liebowitz, M. R. (1987). Social Phobia. Modern Problems of Pharmacopsychiatry, 22, 141-173.

Liu, C., \& Kuo, F. (2007). A study of Internet addiction through the lens of the interpersonal theory. Cyberpsychology \& Behavior, 10, 799-804.

Montag, C., Flierl, M., Markett Walter, S., Jurkiewicz, N., Reuter, M. (2011). Internet addiction and personality in firstperson shooter video gamers. Journal of Media Psychology, 23, 163-173.

Morrison, C. M., \& Gore, H. (2010). The relationship between excessive Internet use and depression: a questionnaire based study of 1,319 young people and adults. Psychopathology, 43, 121-126.

Pontes, H. M., Szabo, A. \& Griffiths, M. D. (2015). The impact of Internet-based specific activities on the perceptions of Internet addiction, quality of life, and excessive usage: A cross-sectional study. Addictive Behaviors Reports, 19-25.

Steinberg, L., \& Monahan, K. C. (2007). Age differences in resistance to peer influence. Developmental Psychology, 43, $1531-1543$

Wangberg, S., Andreassen, H., Prokosch, H., Mariavogos Santana, S., Sørensen, T., \& Chronaki, C. (2007). Relations between Internet use, socio-economic status (SES), social support and subjective health. Health Promotion International, 23, $70-77$.

Wei, H. T., Chen, M. U., Huang, P. C., \& Bai, Y. M. (2012). The association between online gaming, social phobia, and depression: an internet survey. BMC Psychiatry, 12, 92.

Weinstein, A., Dorani, D., Elhadif, R., Bukovza, Y., \& Yarmulnik, A. (2015). Internet addiction is associated with social anxiety in young adults," Annals of Clinical Psychiatry, 27 (1), 1-7.

World Vision. (2014). A study on child online safety in Albania. Retrieved from http://www.wvi.org/albania/publication/study-child-online-safety-albania

Yen, J. Y., Ko, C. H., Yen, C. F., Wu, H. Y., \& Yang, M. J. (2007). The comorbid psychiatric symptoms of Internet addiction: attention deficit and hyperactivity disorder (ADHD), depression, social phobia, and hostility. Journal of Adolescent Health, 41, 93-98.

Young, K. S. (2015). The evolution of internet addiction. (pp. 3-17). In: C. Montag \& M. Reute, Internet addiction: Neuroscientific approaches and therapeutical interventions. Germany: Springer

Zhou, N., \& Fang, X. (2015). Beyond peer contagion: Unique and interactive effects of multiple peer influences on Internet addiction among Chinese adolescents. Computers in Human Behavior, 50, 231-238. 